\title{
Effect of Dietary Vegetable Oils on the Growth Performance and Fatty Acid Composition of Fingerlings of Rainbow Trout,Oncorhynchus mykiss
}

\author{
Ali Masiha ${ }^{1}$, Eisa Ebrahimi ${ }^{1, *}$, Nasrollah Mahboobi Soofiani ${ }^{1}$, Mahdi Kadivar ${ }^{2}$ \\ ${ }^{1}$ Division of Fisheries, Department of Natural Resources, Isfahan University of Technology, Isfahan, 84156-83111, Iran \\ ${ }^{2}$ Department of Food science, College of Agriculture, Isfahan University of Technology, Isfahan, 84156-83111, Iran \\ *Corresponding Author: e_ebrahimi@cc.iut.ac.ir
}

Copyright $(2013$ Horizon Research Publishing All rights reserved.

\begin{abstract}
This study evaluated the suitability of canola and flaxseed oils as source of supplemental dietary lipid for fingerlings of rainbow trout. Triplicate groups of the 30 fingerlings were fed twice daily by iso-nitrogenous, and iso-calorific diets for 8 weeks. Experimental diets consisted of $30.2 \%$ protein, $18.6 \mathrm{~kJ} / \mathrm{g}$ energy and $16.6 \%$ lipid from fish oil, canola oil, flaxseed oil, 1:1 blends of fish and canola oils, 1:1 blends of fish and flaxseed oils, 1:1 blends of canola and flaxseed oils and 1:1:1 blends of fish, canola and flaxseed oils. Protein and lipid was significantly highest in fish fed the fish and canola oils diet. Condition factor of fingerlings reared on fish oil diet were significantly higher than other treatments. Protein efficiency ratio (PER) was significantly higher in fish fed the flaxseed oil diet and canola and flaxseed oils diet. The results of fatty acid profile revealed that flesh fatty acid composition is effected by dietary fatty acid composition. The highest amounts of HUFA n-3 were detected in fish fed fish oil diet, which was significantly different from other treatments. Present results indicate the fingerlings can be reared on diets in which fish oil has been replaced with canola and flaxseed oils.
\end{abstract}

Keywords Rainbow trout; Canola oil; flaxseed oil; Fatty acid; Body composition

\section{Introduction}

In the course of just a few decades, fish farming has developed into a highly productive and efficient industry for the production of animal protein for human consumption. In addition to good growing conditions, a prerequisite for productivity and economic sustainability in fish farming is a reliable supply of effective feeds. For various reasons, fish meal and fish oil have historically been the dominant raw materials in the production of fish feeds. Due to the development of more energy dense feed types together with the general growth of the aquaculture industry, a significant proportion of the total global production of fish oil is used for fish feed production. A lipid requirement equal to $100 \%$ of the world's total fish oil production is estimated by the year 2010 [1].

While marine oils are superior in their fatty acid composition, they also contain a variety of toxic compounds including polychlorinated dibenzo-p-dioxins (PCDD), polychlorinated dibenzofurans (PCDF) and dioxin-like polychlorinated biphenyls (DL-PCB), particularly the non-ortho and mono-ortho substituted PCBs [2-5]. These compounds are carcinogenic and immunosuppressive in humans [6-8].

It is well-known that lipid oxidation is one of the major problems in fish-derived food products. Polyunsaturated fatty acids (PUFA) are more easily oxidized than saturated fatty acids (SFA), and therefore, food products enhanced with the PUFA n-3 are also more prone to lipid oxidation. There are potential human health risks associated with increased consumption of oxidized PUFA n-3 products $[9,10]$. Another important factor to limit a more common use of PUFA n-3 enhanced food products is the development of off-flavors due to the lipid oxidation that may be offensive to consumers [11].

While it is obvious that a substitute must be found, replacing fish oil in aquaculture diets presents difficulties because most vegetable oils are relatively poor sources of n-3 fatty acids. Exceptions to this are flaxseed and canola oils which are rich in alpha linolenic acid (18:3n-3) (53\% and $12 \%$ respectively) [12]. However, these oils are devoid of longer chain n-3 highly unsaturated fatty acids (HUFA n-3) and their inclusion in trout diets results in a significant decrease in the tissue levels of eicosapentaenoic acid (20:5n-3; EPA) and docosahexaenoic acid (22:6n-3; DHA) $[13,14]$. Moreover, enhancement of omega-3 fatty acid content in rainbow trout fillet was observed in farmed rainbow trout and brook trout (Salvelinusfontinalis) as results of flaxseed oil inclusion in diet $[15,16]$. 
Freshwater fish are capable of converting C18 PUFA to the longer chain C20 and C22 PUFA [17] which are the functionally essential fatty acids in vertebrates [18].

Several studies conducted on freshwater fish indicated that vegetable oils can successfully replace fish oil in fish feeds without affecting survival and growth [19,20]. Caballero et al. (2002) [21] reported that in rainbow trout up to $80-90 \%$ of vegetable oils (e.g., soybean; rapeseed; olive; and palm oils) can be used without compromising their growth. It has also been reported that partial replacement of fish oil by vegetable oils such as rapeseed, soybean, flaxseed or palm oils in fish feeds has no negative impacts on growth and survival of Atlantic salmon (Salmosalar) [22], brook char (Salvelinusfontinalis) [23], gilthead sea bream (Sparusaurata) and European seabass (Dicentrarchuslabrax) [24] and rainbow trout $[21,25]$.

\section{Objectives}

The aim of the present study was to evaluate the effects of fish oil replacement with canola and flaxseed oils (relatively easily obtained and low priced oil) on growth, feed conversion ratio and fillet fatty acid composition of fingerlings of rainbow trout.

\section{Methods}

Six hundred and thirty individual fish with mean initial body weight of $16.49 \pm 0.46 \mathrm{~g}$, rainbow trout fingerlings, were purchased from CheshmehDimeh fish hatchery (ShahreKord, Chaharmahal and Bakhtiari, Iran) and used in this study. Prior to the start of the experiment, fish were acclimatized to the new environmental conditions and the commercial diet (SFT2 of Chine food production factory, Tehran, Iran) for a two week period within a semi recirculating system.

\subsection{Experimental Diets}

Seven iso-nitrogenous, iso-calorific and iso-lipidic purified experimental diets were formulated from 100\% fish oil (FO), 100\% canola oil (CO), 100\% flaxseed oil (FxO), 1:1 blends of fish and canola oils (FCO), 1:1 blends of fish and flaxseed oils (FFxO), 1:1 blends of canola and flaxseed oils $(\mathrm{CFxO})$ and 1:1:1 blends of fish, canola and flaxseed oils (FCFxO) (Table 1). The nutritional content of the diets are presented in table 1 . Diets were prepared and stored as reported previously [26,27].

\subsection{Chemical Analysis}

Fish allocated for flesh analysis were filleted (denuded of skin and bone) and stored at $-20^{\circ} \mathrm{C}$ until used for fillet proximate analysis and Fish allocated for fatty acids analysis were stored at $-80^{\circ} \mathrm{C}$ until analyzed for fatty acids. Proximate analysis was conducted using standard procedures [28], percentage moisture (dried at $80^{\circ} \mathrm{C}$ to constant weight), protein (Kjeldahl nitrogen; $\mathrm{N} \times 6.25$ ) in an automated Kjeltech (Model 2300, Tecator, Sweden), total lipid by chloroform/methanol extraction $(2: 1$, by vol) [29] as modified by Ways and Hanahan (1964) [30] and ash by incineration in a muffle furnace (Model WIT, C \&LTetlow, Australia) at $550^{\circ} \mathrm{C}$ for $18 \mathrm{~h}$. Fatty acid analysis was carried out on each of the added dietary oils, experimental diets and fillet samples from each of the replicates. Fatty acid methyl esters (FAME) were prepared from aliquots of total lipids by acid catalyzed transmethylation with sulfuric acid in methanol overnight at $50^{\circ} \mathrm{C}$ [31]. FAME were purified by TLC using hexane/diethyl ether/acetic acid (85:15:15, by vol) as solvent [32]. Separation of FAME was carried out in Gas Chromatograph system (Agilent Technologies, 6890N, USA) equipped with a flame ionization detector (FID), a crosslinked silica capillary column HP-88 (100 m, $250 \mu \mathrm{m}$ ID, $0.2 \mu \mathrm{m}$ film thickness), on-column injection and using Helium as carrier gas. The column was programmed for an initial temperature of $140^{\circ} \mathrm{C}$ held for $5 \mathrm{~min}$, rising at a rate of $4^{\circ} \mathrm{C} / \mathrm{min}$ to the final temperature $240^{\circ} \mathrm{C}$ and held for $10 \mathrm{~min}$. Injector and detector temperatures were $230^{\circ} \mathrm{C}$ and $260^{\circ} \mathrm{C}$, respectively. Helium was used as the carrier gas with flow rate of $1.1 \mathrm{~mL} / \mathrm{min}$. The flow rates of compressed air and hydrogen were $300 \mathrm{~mL} / \mathrm{min}$ and $30 \mathrm{~mL} / \mathrm{min}$, respectively. Identification and quantification of FAME were based on the comparison of the sample retention time with known standards (Sigma Chemicals, St. Louis, USA).

\subsection{Statistical Analysis}

Mean values and standard deviation for each parameter measured for all treatments were first calculated. The results were subjected to one-way ANOVA to test the effect of vegetable oils blend replacement on fish performance. Data were analyzed using statistical packages SPSS v15 (SPSS Inc., Chicago, IL, USA). Differences between means were compared using Duncan's multiple range test at significance of differences $(P<0.05)$ among dietary treatments. Linear regression analyses were performed between dietary and fillet fatty acid concentrations.

\section{Results}

\subsection{Diet Composition}

The proximate compositions and energy content of the experimental diets are presented in Table 1.

The fish oil diet (FOD) contained the highest level of SFA $(33.32 \%)$ predominantly in the form of palmitic acid (16:0) and stearic acid (18:0) which accounted for $(22.71 \%)$ and $(5.85 \%)$, respectively (Table 2$)$. Monounsaturated fatty acids (MUFA) concentrations were highest in the canola oil diet (COD) (64.57\%), represented mainly as oleic acid (18:1n-9, $59.87 \%)$. The FOD was richest in PUFA (n-6+n-3) (18.63\%) with $\alpha$-linolenic acid $(18: 3 n-3,4.58 \%)$ and linoleic acid $(18: 2 \mathrm{n}-6,0.42 \%)$ as the principal fatty acids. The highest levels of EPA and DHA were in the FOD, with $2.95 \%$ and $6.65 \%$, respectively. Fatty acids of the n-3 and n-6 series were observed in highest concentration in the FOD, which accounted for $(15.75 \%)$ and $(2.88 \%)$, respectively. Levels of HUFA n-3 were found in highest concentrations in the FOD with $9.60 \%$. 
Table 1. Ingredient (\%), proximate composition (\% wet weight) and energy $(\mathrm{kJ} / \mathrm{g})$ of the experimental diets

\begin{tabular}{|c|c|c|c|c|c|c|c|}
\hline Diet components & FOD & COD & FxOD & FCOD & FFxOD & CFxOD & FCFxOD \\
\hline Fish Meal & 58 & 58 & 58 & 58 & 58 & 58 & 58 \\
\hline Soybean Meal & 20 & 20 & 20 & 20 & 20 & 20 & 20 \\
\hline Wheat Meal & 8.6 & 8.6 & 8.6 & 8.6 & 8.6 & 8.6 & 8.6 \\
\hline Fish Oil & 8 & 0 & 0 & 4 & 4 & 0 & 2.67 \\
\hline Canola oil & 0 & 8 & 0 & 4 & 0 & 4 & 2.67 \\
\hline Flaxseed Oil & 0 & 0 & 8 & 0 & 4 & 4 & 2.67 \\
\hline Vitamin premix ${ }^{a}$ & 2 & 2 & 2 & 2 & 2 & 2 & 2 \\
\hline Mineral premix ${ }^{b}$ & 1.5 & 1.5 & 1.5 & 1.5 & 1.5 & 1.5 & 1.5 \\
\hline Lysine & 0.07 & 0.07 & 0.07 & 0.07 & 0.07 & 0.07 & 0.07 \\
\hline Methionine & 0.13 & 0.13 & 0.13 & 0.13 & 0.13 & 0.13 & 0.13 \\
\hline Choline chloride & 0.2 & 0.2 & 0.2 & 0.2 & 0.2 & 0.2 & 0.2 \\
\hline Molasses & 1 & 1 & 1 & 1 & 1 & 1 & 1 \\
\hline Salt & 0.5 & 0.5 & 0.5 & 0.5 & 0.5 & 0.5 & 0.5 \\
\hline \multicolumn{8}{|c|}{ Proximate composition } \\
\hline Moisture & $9.80 \pm 0.58$ & $10.20 \pm 0.37$ & $9.10 \pm 0.56$ & $9.40 \pm 0.48$ & $9.63 \pm 0.38$ & $10.09 \pm 0.25$ & $9.58 \pm 0.45$ \\
\hline Ash & $14.59 \pm 0.31$ & $14.19 \pm 0.37$ & $14.76 \pm 0.45$ & $15.29 \pm 0.30$ & $15.29 \pm 0.27$ & $14.86 \pm 0.36$ & $15.12 \pm 0.38$ \\
\hline Crude protein & $30.66 \pm 0.47$ & $30.26 \pm 0.45$ & $30.15 \pm 0.42$ & $29.97 \pm 0.39$ & $29.85 \pm 0.48$ & $30.06 \pm 0.25$ & $30.47 \pm 0.32$ \\
\hline Crude lipid & $16.09 \pm 0.29$ & $16.89 \pm 0.18$ & $16.52 \pm 0.38$ & $17.06 \pm 0.31$ & $16.81 \pm 0.35$ & $16.74 \pm 0.42$ & $16.32 \pm 0.36$ \\
\hline Crude fiber & $2.16 \pm 0.12$ & $2.31 \pm 0.18$ & $2.42 \pm 0.16$ & $1.95 \pm 0.21$ & $2.01 \pm 0.23$ & $2.23 \pm 0.18$ & $2.09 \pm 0.22$ \\
\hline $\mathrm{NFE}^{\mathrm{c}}$ & 26.70 & 26.15 & 27.05 & 26.33 & 26.41 & 26.02 & 26.42 \\
\hline Energy $^{\mathrm{d}}$ & 18.56 & 18.71 & 18.71 & 18.68 & 18.57 & 18.57 & 18.54 \\
\hline
\end{tabular}

Diet abbreviations, FOD: 100\% fish oil; COD: 100\% canola oil; FxOD: 100\% flaxseed oil; FCOD: 1:1 blends of fish and canola oils; FFxOD: 1:1 blends of fish and flaxseed oils; CFxOD: 1:1 blends of canola and flaxseed oils; FCFxOD: 1:1:1 blends of fish, canola and flaxseed oils.

aContains (mg/kg food): E (30), K (3), niacin (40), thiamine (2), riboflavin (7), pyridoxine (3), folacin (1.5), pantothenic acid (18), biotin (0.7) and cyanocobalamin (0.18).

bContains (mg/kg food): $\mathrm{Mg}(100), \mathrm{Zn}(60), \mathrm{Fe}(40), \mathrm{Cu}(5), \mathrm{Co}(0.1), \mathrm{I}(1)$ and Antioxidant (100).

cNFE: nitrogen free extract, calculated by difference (100 - moisture - ash - crude protein - crude lipid - crude fibers).

dCalculated on the basis of $23.6,39.5$ and $17.2 \mathrm{~kJ} / \mathrm{g}$ of protein, fat and carbohydrate, respectively. 
Table 2. Fatty acid composition (percentage of total fatty acids) of the oils and experimental diets.

\begin{tabular}{|c|c|c|c|c|c|c|c|c|c|c|}
\hline & Fish Oil & $\begin{array}{c}\text { Canola } \\
\text { Oil } \\
\end{array}$ & $\begin{array}{c}\text { Flaxseed } \\
\text { Oil } \\
\end{array}$ & FOD & COD & FxOD & FCOD & FFxOD & CFxOD & FCFxOD \\
\hline $14: 0$ & 0.06 & - & - & 0.07 & 0.04 & 0.04 & 0.04 & 0.05 & 0.04 & 0.04 \\
\hline $15: 0$ & 0.32 & - & - & 0.23 & 0.08 & 0.09 & 0.14 & 0.17 & 0.08 & 0.12 \\
\hline $16: 0$ & 20.73 & 5.77 & 6.79 & 22.71 & 14.33 & 16.48 & 17.41 & 19.80 & 14.37 & 17.45 \\
\hline $17: 0$ & 0.72 & 0.07 & 0.11 & 0.72 & 0.41 & 0.51 & 0.56 & 0.67 & 0.43 & 0.58 \\
\hline $18: 0$ & 4.16 & 1.27 & 4.48 & 5.85 & 4.46 & 6.04 & 5.08 & 6.20 & 5.13 & 5.25 \\
\hline 19:0 & 2.89 & 3.78 & 1.23 & 2.94 & 3.13 & 2.31 & 3.04 & 2.53 & 2.61 & 2.65 \\
\hline $21: 0$ & 0.18 & 0.21 & - & 0.25 & 0.16 & 0.24 & 0.19 & 0.29 & 0.21 & 0.18 \\
\hline $22: 0$ & 0.24 & 0.14 & 0.04 & 0.20 & 0.13 & 0.13 & 0.20 & 0.15 & 0.14 & 0.14 \\
\hline $23: 0$ & 0.21 & 0.57 & 0.57 & 0.22 & 0.37 & 0.30 & 0.33 & 0.33 & 0.46 & 0.35 \\
\hline $24: 0$ & 0.18 & - & - & 0.14 & 0.07 & 0.08 & 0.13 & 0.11 & 0.08 & 0.10 \\
\hline SFA & 29.68 & 11.80 & 13.22 & 33.32 & 23.17 & 26.22 & 27.12 & 30.29 & 23.56 & 26.86 \\
\hline $14: 1$ & 3.77 & 0.19 & 0.26 & 3.11 & 1.31 & 1.37 & 2.07 & 2.26 & 1.31 & 2.00 \\
\hline $15: 1$ & 0.80 & 0.04 & 0.07 & 0.71 & 0.34 & 0.38 & 0.51 & 0.56 & 0.34 & 0.50 \\
\hline $16: 1 n-7$ & 5.24 & 0.34 & 0.34 & 4.92 & 2.54 & 2.59 & 3.61 & 3.86 & 2.47 & 3.44 \\
\hline $17: 1$ & 0.71 & - & 0.06 & 0.65 & 0.26 & 0.27 & 0.41 & 0.41 & 0.21 & 0.32 \\
\hline 18:1n-9 & 33.57 & 77.33 & 34.19 & 38.79 & 59.87 & 41.75 & 49.63 & 40.50 & 49.79 & 45.90 \\
\hline $24: 1 n-9$ & 0.44 & - & - & 0.41 & 0.24 & 0.14 & 0.39 & 0.26 & 0.27 & 0.33 \\
\hline MUFA & 44.52 & 77.91 & 34.92 & 48.59 & 64.57 & 46.50 & 56.61 & 47.85 & 54.39 & 52.49 \\
\hline $18: 2 n-6$ & 0.37 & 1.01 & 0.38 & 0.42 & 0.70 & 0.39 & 0.47 & 0.61 & 0.57 & 0.50 \\
\hline $18: 3 n-6$ & 0.05 & 0.38 & - & 0.04 & 0.21 & 0.05 & 0.14 & 0.05 & 0.14 & 0.10 \\
\hline $20: 2 n-6$ & 2.48 & 0.14 & 0.13 & 1.21 & 0.29 & 0.24 & 0.82 & 0.68 & 0.28 & 0.64 \\
\hline $20: 3 n-6$ & 0.18 & - & - & 0.20 & 0.22 & 0.25 & 0.28 & 0.21 & 0.23 & 0.21 \\
\hline $20: 4 n-6$ & 0.02 & - & - & 0.06 & 0.04 & 0.03 & 0.04 & 0.05 & 0.05 & 0.04 \\
\hline $22: 2 n-6$ & 0.72 & - & - & 0.65 & 0.40 & 0.37 & 0.62 & 0.52 & 0.44 & 0.55 \\
\hline $22: 5 n-6$ & 0.36 & - & - & 0.30 & 0.16 & 0.18 & 0.28 & 0.21 & 0.17 & 0.24 \\
\hline PUFA n-6 & 4.18 & 1.53 & 0.50 & 2.88 & 2.03 & 1.51 & 2.65 & 2.32 & 1.89 & 2.28 \\
\hline $18: 3 n-3$ & 2.07 & 7.05 & 51.36 & 4.58 & 4.87 & 21.93 & 3.95 & 12.64 & 14.79 & 10.88 \\
\hline $18: 4 n-3$ & 0.32 & 1.71 & - & 0.60 & 1.25 & 0.71 & 0.87 & 0.84 & 1.05 & 0.85 \\
\hline $20: 3 n-3$ & 0.05 & - & - & 0.61 & 0.04 & 0.05 & 0.07 & 0.05 & 0.06 & 0.05 \\
\hline $20: 5 n-3$ & 5.90 & - & - & 2.95 & 0.96 & 0.73 & 2.29 & 1.69 & 0.99 & 1.78 \\
\hline $22: 5 n-3$ & 0.48 & - & - & 0.36 & 0.21 & 0.22 & 0.32 & 0.28 & 0.22 & 0.27 \\
\hline $22: 6 n-3$ & 12.82 & - & - & 6.65 & 2.91 & 2.16 & 5.82 & 4.05 & 3.07 & 4.59 \\
\hline PUFA n-3 & 21.62 & 8.76 & 51.36 & 15.75 & 10.24 & 25.80 & 13.32 & 19.55 & 20.17 & 18.41 \\
\hline HUFA n-3 & 18.72 & - & - & 9.60 & 3.87 & 2.89 & 8.11 & 5.74 & 4.06 & 6.37 \\
\hline
\end{tabular}

not detected.

See Table 1 for diet abbreviations.

Table 3.. Mean $( \pm \mathrm{SD})$ of growth, feed utilization and other body parameters of rainbow trout reared on the experimental diets.

\begin{tabular}{|c|c|c|c|c|c|c|c|}
\hline & FOD & COD & FxOD & FCOD & FFxOD & CFxOD & FCFxOD \\
\hline MIBW & $16.12 \pm 0.27$ & $16.44 \pm 0.30$ & $16.30 \pm 0.78$ & $16.82 \pm 0.11$ & $16.74 \pm 0.33$ & $16.47 \pm 0.81$ & $16.55 \pm 0.21$ \\
\hline MFBW & $58.05 \pm 6.98$ & $58.50 \pm 7.96$ & $56.56 \pm 8.05$ & $54.74 \pm 12.22$ & $58.52 \pm 14.58$ & $56.98 \pm 7.62$ & $58.05 \pm 6.98$ \\
\hline CF & $1.22 \pm 0.10^{\mathrm{a}}$ & $1.18 \pm 0.11^{\mathrm{ab}}$ & $1.15 \pm 0.11^{\mathrm{b}}$ & $1.19 \pm 0.11^{\mathrm{ab}}$ & $1.12 \pm 0.10^{\mathrm{b}}$ & $1.14 \pm 0.11^{\mathrm{b}}$ & $1.14 \pm 0.07^{\mathrm{b}}$ \\
\hline SGR & $2.29 \pm 0.02$ & $2.27 \pm 0.02$ & $2.22 \pm 0.06$ & $2.10 \pm 0.10$ & $2.23 \pm 0.08$ & $2.22 \pm 0.03$ & $2.18 \pm 0.13$ \\
\hline WG & $260.09 \pm 4.25$ & $255.89 \pm 4.07$ & $246.71 \pm 10.78$ & $224.56 \pm 17.82$ & $249.54 \pm 15.23$ & $245.74 \pm 6.52$ & $240.19 \pm 24.37$ \\
\hline FCR & $0.90 \pm 0.17$ & $0.97 \pm 0.17$ & $1.01 \pm 0.05$ & $1.04 \pm 0.54$ & $0.99 \pm 0.30$ & $1.07 \pm 0.41$ & $1.01 \pm 0.21$ \\
\hline SR & $100.00 \pm 0.00$ & $96.67 \pm 5.77$ & $100.00 \pm 0.00$ & $90.00 \pm 17.32$ & $100.00 \pm 0.00$ & $93.33 \pm 5.77$ & $100.00 \pm 0.00$ \\
\hline HIS & $1.21 \pm 0.13$ & $1.16 \pm 0.08$ & $1.14 \pm 0.06$ & $1.11 \pm 0.10$ & $1.15 \pm 0.06$ & $1.12 \pm 0.10$ & $1.22 \pm 0.14$ \\
\hline PER & $1.92 \pm 0.22^{\mathrm{bc}}$ & $2.24 \pm 0.17^{\mathrm{b}}$ & $3.12 \pm 0.31^{\mathrm{a}}$ & $1.49 \pm 0.49^{\mathrm{c}}$ & $2.37 \pm 0.16^{\mathrm{b}}$ & $2.86 \pm 0.22^{\mathrm{a}}$ & $1.68 \pm 0.16^{\mathrm{c}}$ \\
\hline
\end{tabular}

Values in the same row with the same superscripts are not significantly different $(P>0.05)$.

See Table 1 for diet abbreviations.

MIBW (g): Mean initial body weight, MFBW (g): Mean final body weight.

$\mathrm{CF}$ : Condition factor $=100 \times($ final weight $(\mathrm{g})) \times(\text { fork length }(\mathrm{cm}))^{-3}$.

SGR $(\% /$ day $)$ : Specific growth rate $=[\operatorname{Ln}($ final weight $)-\operatorname{Ln}($ initial weight $)] \times(\text { number of days })^{-1} \times 100$.

WG $(\%)$ : Weight Gain $=($ final weight - initial weight $) \times(\text { initial weight })^{-1} \times 100$.

FCR: Feed conversion ratio $=($ dry feed fed $) \times(\text { wet weight gain })^{-1}$.

SR (\%): Survival rate=number of fish in each group remaining on day 56 -(initial number of fish $)^{-1} \times 100$

HIS (\%): Hepatosomatic index $=($ weight of liver $) \times(\text { total fish weight })^{-1} \times 100$.

PER: Protein efficiency ratio $=($ final weight - initial weight $) \times(\text { mass of protein fed })^{-1}$. 


\subsection{Growth}

The overall mortality was low and did not appear to be related to the dietary treatment (Table 3). The mean final body weight (MFBW) of fingerlings of rainbow trout ranged from $54.7 \pm 12.2$ (fish and canola oil diet) (FCOD)) to $58.5 \pm 14.6$ (fish and flaxseed oil diet) (FFxOD)). The differences between the MFBW of fish receiving different diets were not significant. Similarly, no significant differences were observed between specific growth rate (SGR), weight gain (WG), feed conversion ratio (FCR), survival rate (SR) and hepatosomatic index (HSI). The condition factor (CF) of fingerlings of rainbow trout was highest in fish fed the FOD and significantly $(\mathrm{P}<0.05)$ higher than those fish fed the flaxseed oil diet (FxOD), FFxOD, canola and flaxseed oil diet (CFxOD) and fish, canola and flaxseed oil diet (FCFxOD). The protein efficiency ratio (PER) was highest in fish fed the FxOD and significantly $(\mathrm{P}<0.05)$ higher than other treatments without treatment 6 (CFxOD) (Table 3).

\subsection{Fillet Proximate Composition}

Results of the proximate analysis of fillet of fish receiving the different dietary treatments are tabulated in Table 4. No significant differences between percent moisture and ash content of fish fed the experimental diets were observed, but the percent of protein and lipid content was highest in fish fed the FCOD which was significantly $(P<0.05)$ higher than those fish fed the FFxOD and FCFxOD and COD, FFxOD, CFxOD and FCFxOD, respectively.

\subsection{Fillet Fatty Acid Composition}

The major fatty acid classes (SFA; MUFA and PUFA) found in the highest concentration werepalmitic, oleic, $\alpha$-linolenic acids along with DHA, respectively (Table 5). The level of SFA was observed in higher $(P<0.05)$ concentrations for fish fed the FOD. Levels of MUFA ranged from $47.40 \pm 0.52$ (FxOD) to $63.28 \pm 0.24$ (COD) and were observed to be significantly highest in fish fed the COD. The fillet of fish fed with the COD and FxOD were particularly rich with in oleic acid $(58.82 \pm 0.86 \%)$ and $\alpha$-linolenic acid $(19.35 \pm 0.40 \%)$, respectively. DHA and arachidonic acid levels were found in higher concentrations in the fillet than in the diets. The highest observed level of EPA and DHA was found in fish fed the FOD $(P<0.05)$. However, DHA was found in high concentrations within all of the dietary treatments, ranging from $5.04 \pm 0.19 \%$ (COD) to $10.73 \pm 0.40 \%$ (FOD). Level of n-3 fatty acids was higher in the fillet than the diet for each of the treatments without treatment 6 (CFxOD) and 7 (FCFxOD), but level of n- 6 fatty acids was higher in the fillet than the diet for each of the treatments without treatment 1 (FOD) and 4 (FCOD), with $\mathrm{n}-6 / \mathrm{n}-3$ ratio ranging from $0.12 \pm 0.00$ to $0.23 \pm 0.02$ in the fillet. The highest HUFA n-3 concentrations $(P<0.05)$ were found in fish fed the FOD $(12.80 \pm 0.45 \%)$, while the lowest value was observed in fish fed the COD $(5.63 \pm 0.22 \%)$.

Regression analysis was used to identify dose response relationship between dietary and fillet fatty acids. As reported in Table 6 , most of the fatty acid concentrations in the fillet were linearly correlated to the dietary fatty acid concentrations.

Table 4. Fillet proximate compositions ( $m e a n \pm S D)$ of rainbow trout reared on different diets, $(\%$ wet weight $)$

\begin{tabular}{|c|c|c|c|c|c|c|c|c|}
\hline & \multirow{2}{*}{ Initial $^{\mathrm{a}}$} & FOD & COD & FxOD & FCOD & FFxOD & CFxOD & FCFxOD \\
\hline Moisture & $78.44 \pm 0.61$ & $76.96 \pm 0.20$ & $76.97 \pm 0.60$ & $76.48 \pm 1.02$ & $76.43 \pm 0.17$ & $77.39 \pm 0.41$ & $77.08 \pm 0.28$ & $77.21 \pm 0.26$ \\
\hline Ash & $1.34 \pm 0.03$ & $1.28 \pm 0.05$ & $1.26 \pm 0.04$ & $1.36 \pm 0.06$ & $1.29 \pm 0.08$ & $1.28 \pm 0.08$ & $1.19 \pm 0.09$ \\
\hline Protein & $15.29 \pm 0.81$ & $17.77 \pm 0.16^{\mathrm{ab}}$ & $18.70 \pm 1.02^{\mathrm{a}}$ & $18.55 \pm 1.15^{\mathrm{a}}$ & $18.95 \pm 0.24^{\mathrm{a}}$ & $16.49 \pm 1.30^{\mathrm{b}}$ & $17.62 \pm 0.73^{\mathrm{ab}}$ & $16.16 \pm 0.90^{\mathrm{b}}$ \\
\hline & & & & & & & & \\
\hline
\end{tabular}

See Table 1 for diet abbreviations.

${ }^{\text {a }}$ Statistics not performed on the initial sample.

Values in the same row with the same superscripts are not significantly different $(\mathrm{P}>0.05)$. 
Table 5. Fillet fatty acid composition (percentage of total fatty acids) of rainbow trout reared on the different diets (mean $\pm \mathrm{SD}$ ).

\begin{tabular}{|c|c|c|c|c|c|c|c|c|}
\hline & Initial & FOD & COD & FxOD & FCOD & FFxOD & CFxOD & FCFxOD \\
\hline $14: 0$ & 0.03 & $0.04 \pm 0.01$ & $0.04 \pm 0.01$ & $0.03 \pm 0.00$ & $0.04 \pm 0.01$ & $0.03 \pm 0.01$ & $0.03 \pm 0.01$ & $0.04 \pm 0.01$ \\
\hline $15: 0$ & 0.09 & $0.16 \pm 0.01^{\mathrm{a}}$ & $0.06 \pm 0.00^{\mathrm{d}}$ & $0.07 \pm 0.01^{\mathrm{d}}$ & $0.10 \pm 0.02^{\mathrm{bc}}$ & $0.10 \pm 0.01^{\mathrm{b}}$ & $0.06 \pm 0.01^{\mathrm{d}}$ & $0.09 \pm 0.01^{\mathrm{c}}$ \\
\hline $16: 0$ & 10.11 & $17.45 \pm 0.29^{\mathrm{a}}$ & $12.85 \pm 0.28^{\mathrm{d}}$ & $12.46 \pm 0.12^{\mathrm{d}}$ & $14.13 \pm 0.39^{b c}$ & $14.42 \pm 0.26^{\mathrm{b}}$ & $13.05 \pm 0.15^{\mathrm{d}}$ & $13.71 \pm 0.62^{\mathrm{c}}$ \\
\hline $17: 0$ & 0.14 & $0.50 \pm 0.01^{\mathrm{a}}$ & $0.32 \pm 0.04^{\mathrm{cd}}$ & $0.15 \pm 0.02^{\mathrm{e}}$ & $0.36 \pm 0.01^{\mathrm{b}}$ & $0.37 \pm 0.00^{\mathrm{b}}$ & $0.29 \pm 0.00^{\mathrm{d}}$ & $0.34 \pm 0.02^{\mathrm{bc}}$ \\
\hline $18: 0$ & 2.90 & $4.46 \pm 0.10^{\mathrm{ab}}$ & $3.78 \pm 0.09^{\mathrm{e}}$ & $4.59 \pm 0.07^{\mathrm{a}}$ & $3.90 \pm 0.16^{\mathrm{de}}$ & $4.27 \pm 0.06^{\mathrm{bc}}$ & $4.20 \pm 0.05^{\mathrm{c}}$ & $4.09 \pm 0.20^{\mathrm{cd}}$ \\
\hline 19:0 & 3.07 & $3.72 \pm 0.45^{\mathrm{ab}}$ & $3.76 \pm 0.09^{\mathrm{a}}$ & $2.10 \pm 0.01^{\mathrm{e}}$ & $3.41 \pm 0.11^{\mathrm{bc}}$ & $2.94 \pm 0.10^{\mathrm{d}}$ & $2.94 \pm 0.10^{\mathrm{d}}$ & $3.15 \pm 0.05^{\mathrm{cd}}$ \\
\hline $21: 0$ & 0.21 & $0.19 \pm 0.06^{\mathrm{b}}$ & $0.18 \pm 0.04^{b}$ & $0.26 \pm 0.02^{\mathrm{a}}$ & $0.18 \pm 0.02^{\mathrm{b}}$ & $0.19 \pm 0.04^{\mathrm{b}}$ & $0.20 \pm 0.01^{\mathrm{b}}$ & $0.18 \pm 0.03^{\mathrm{b}}$ \\
\hline $22: 0$ & 0.77 & $0.55 \pm 0.06^{\mathrm{c}}$ & $0.93 \pm 0.09^{\mathrm{a}}$ & $0.56 \pm 0.08^{\mathrm{c}}$ & $0.71 \pm 0.05^{\mathrm{b}}$ & $0.56 \pm 0.04^{\mathrm{c}}$ & $0.67 \pm 0.03^{\mathrm{b}}$ & $0.63 \pm 0.02^{\mathrm{bc}}$ \\
\hline $23: 0$ & 0.72 & $0.28 \pm 0.02^{\mathrm{g}}$ & $0.49 \pm 0.03^{\mathrm{e}}$ & $0.90 \pm 0.08^{\mathrm{a}}$ & $0.42 \pm 0.02^{\mathrm{f}}$ & $0.67 \pm 0.02^{\mathrm{c}}$ & $0.73 \pm 0.03^{\mathrm{b}}$ & $0.59 \pm 0.02^{\mathrm{d}}$ \\
\hline $24: 0$ & 0.41 & $0.41 \pm 0.03^{\text {cd }}$ & $0.25 \pm 0.03^{\mathrm{e}}$ & $0.72 \pm 0.15^{\mathrm{a}}$ & $0.34 \pm 0.04^{\mathrm{de}}$ & $0.60 \pm 0.03^{\mathrm{ab}}$ & $0.48 \pm 0.04^{\mathrm{bc}}$ & $0.50 \pm 0.06^{\mathrm{bc}}$ \\
\hline SFA & 18.44 & $27.74 \pm 0.53^{\mathrm{a}}$ & $22.66 \pm 0.29^{d}$ & $21.84 \pm 0.20^{\mathrm{e}}$ & $23.56 \pm 0.45^{\mathrm{bc}}$ & $24.15 \pm 0.25^{b}$ & $22.64 \pm 0.02^{\mathrm{d}}$ & $23.33 \pm 0.83^{\mathrm{cd}}$ \\
\hline $14: 1$ & 1.42 & $2.25 \pm 0.08^{\mathrm{a}}$ & $1.18 \pm 0.06^{\mathrm{d}}$ & $1.10 \pm 0.03^{\mathrm{d}}$ & $1.59 \pm 0.01^{\mathrm{b}}$ & $1.56 \pm 0.05^{\mathrm{b}}$ & $1.15 \pm 0.02^{\mathrm{d}}$ & $1.45 \pm 0.02^{\mathrm{c}}$ \\
\hline $15: 1$ & 0.17 & $0.52 \pm 0.02^{\mathrm{a}}$ & $0.27 \pm 0.01^{\mathrm{c}}$ & $0.27 \pm 0.00^{\mathrm{c}}$ & $0.34 \pm 0.03^{\mathrm{b}}$ & $0.36 \pm 0.01^{\mathrm{b}}$ & $0.27 \pm 0.01^{\mathrm{c}}$ & $0.33 \pm 0.02^{\mathrm{b}}$ \\
\hline $16: 1 \mathrm{n}-7$ & 1.77 & $4.49 \pm 0.09^{\mathrm{a}}$ & $2.46 \pm 0.20^{\mathrm{d}}$ & $2.35 \pm 0.03^{\mathrm{d}}$ & $3.20 \pm 0.13^{b}$ & $3.28 \pm 0.09^{\mathrm{b}}$ & $2.36 \pm 0.04^{\mathrm{d}}$ & $3.00 \pm 0.04^{\mathrm{c}}$ \\
\hline $17: 1$ & 0.18 & $0.48 \pm 0.03^{\mathrm{a}}$ & $0.22 \pm 0.05^{\mathrm{de}}$ & $0.17 \pm 0.13^{\mathrm{e}}$ & $0.34 \pm 0.02^{\mathrm{bc}}$ & $0.36 \pm 0.02^{\mathrm{b}}$ & $0.24 \pm 0.00^{\text {cde }}$ & $0.30 \pm 0.05^{\text {bcd }}$ \\
\hline $18: \ln -9$ & 61.25 & $44.85 \pm 0.45^{\mathrm{d}}$ & $58.82 \pm 0.56^{\mathrm{a}}$ & $43.25 \pm 0.63^{\mathrm{e}}$ & $53.91 \pm 0.33^{b}$ & $44.71 \pm 0.15^{\mathrm{d}}$ & $51.21 \pm 0.57^{\mathrm{c}}$ & $50.59 \pm 1.61^{\mathrm{c}}$ \\
\hline $24: 1 n-9$ & 0.10 & $0.40 \pm 0.03^{\mathrm{a}}$ & $0.33 \pm 0.04^{\mathrm{b}}$ & $0.27 \pm 0.05^{\mathrm{c}}$ & $0.35 \pm 0.02^{\mathrm{ab}}$ & $0.32 \pm 0.01^{\mathrm{bc}}$ & $0.30 \pm 0.03^{b c}$ & $0.32 \pm 0.03^{b c}$ \\
\hline MUFA & 64.88 & $52.99 \pm 0.38^{\mathrm{d}}$ & $63.28 \pm 0.24^{\mathrm{a}}$ & $47.40 \pm 0.52^{\mathrm{f}}$ & $59.73 \pm 0.17^{\mathrm{b}}$ & $50.60 \pm 0.02^{\mathrm{e}}$ & $55.53 \pm 0.58^{\mathrm{c}}$ & $56.00 \pm 1.50^{\mathrm{c}}$ \\
\hline $18: 2 n-6$ & 0.71 & $0.41 \pm 0.05^{\mathrm{d}}$ & $0.78 \pm 0.06^{\mathrm{a}}$ & $0.52 \pm 0.09^{c}$ & $0.54 \pm 0.03^{\mathrm{c}}$ & $0.42 \pm 0.02^{\mathrm{d}}$ & $0.66 \pm 0.05^{b}$ & $0.57 \pm 0.02^{\mathrm{c}}$ \\
\hline $18: 3 n-6$ & 0.05 & $0.06 \pm 0.00^{\mathrm{c}}$ & $0.17 \pm 0.01^{\mathrm{a}}$ & $0.06 \pm 0.01^{\mathrm{c}}$ & $0.11 \pm 0.01^{\mathrm{b}}$ & $0.06 \pm 0.02^{\mathrm{c}}$ & $0.12 \pm 0.02^{\mathrm{b}}$ & $0.11 \pm 0.01^{\mathrm{b}}$ \\
\hline $20: 2 n-6$ & 0.88 & $0.89 \pm 0.06^{\mathrm{c}}$ & $0.46 \pm 0.05^{\mathrm{d}}$ & $1.54 \pm 0.12^{\mathrm{a}}$ & $0.60 \pm 0.06^{\mathrm{d}}$ & $1.11 \pm 0.04^{\mathrm{b}}$ & $1.10 \pm 0.14^{\mathrm{b}}$ & $0.93 \pm 0.03^{c}$ \\
\hline $20: 3 n-6$ & 0.13 & $0.09 \pm 0.01^{\mathrm{bc}}$ & $0.12 \pm 0.01^{\mathrm{a}}$ & $0.07 \pm 0.03^{\mathrm{c}}$ & $0.12 \pm 0.02^{\mathrm{ab}}$ & $0.08 \pm 0.02^{\mathrm{c}}$ & $0.11 \pm 0.00^{\mathrm{ab}}$ & $0.11 \pm 0.01^{\mathrm{ab}}$ \\
\hline $20: 4 n-6$ & 0.86 & $0.13 \pm 0.03$ & $0.17 \pm 0.05$ & $0.14 \pm 0.01$ & $0.17 \pm 0.01$ & $0.13 \pm 0.01$ & $0.15 \pm 0.01$ & $0.15 \pm 0.03$ \\
\hline $22: 2 n-6$ & 0.43 & $0.77 \pm 0.07^{\mathrm{a}}$ & $0.69 \pm 0.04^{\mathrm{ab}}$ & $0.65 \pm 0.03^{\mathrm{b}}$ & $0.72 \pm 0.03^{\mathrm{ab}}$ & $0.74 \pm 0.02^{\mathrm{ab}}$ & $0.69 \pm 0.07^{\mathrm{ab}}$ & $0.68 \pm 0.05^{\mathrm{b}}$ \\
\hline $22: 5 n-6$ & 0.33 & $0.24 \pm 0.03^{\mathrm{ab}}$ & $0.24 \pm 0.02^{\mathrm{ab}}$ & $0.20 \pm 0.03^{c}$ & $0.26 \pm 0.02^{\mathrm{a}}$ & $0.22 \pm 0.01^{\mathrm{ab}}$ & $0.22 \pm 0.00^{\mathrm{bc}}$ & $0.23 \pm 0.02^{\mathrm{abc}}$ \\
\hline PUFA n-6 & 3.40 & $2.60 \pm 0.24^{\mathrm{c}}$ & $2.63 \pm 0.17^{\mathrm{c}}$ & $3.18 \pm 0.23^{\mathrm{a}}$ & $2.52 \pm 0.13^{\mathrm{c}}$ & $2.75 \pm 0.02^{\mathrm{c}}$ & $3.06 \pm 0.20^{\mathrm{ab}}$ & $2.77 \pm 0.03^{b c}$ \\
\hline $18: 3 n-3$ & 4.27 & $1.94 \pm 0.07^{\mathrm{g}}$ & $3.23 \pm 0.25^{\mathrm{e}}$ & $19.35 \pm 0.40^{\mathrm{a}}$ & $2.76 \pm 0.03^{f}$ & $10.60 \pm 0.07^{\mathrm{b}}$ & $10.01 \pm 0.10^{c}$ & $7.95 \pm 0.23^{\mathrm{d}}$ \\
\hline $18: 4 n-3$ & 2.61 & $1.04 \pm 0.04^{\mathrm{c}}$ & $1.75 \pm 0.32^{\mathrm{a}}$ & $1.01 \pm 0.03^{\mathrm{c}}$ & $1.48 \pm 0.14^{\mathrm{b}}$ & $1.09 \pm 0.06^{\mathrm{c}}$ & $1.57 \pm 0.06^{\mathrm{ab}}$ & $1.43 \pm 0.14^{\mathrm{b}}$ \\
\hline $20: 3 n-3$ & 0.53 & $0.30 \pm 0.03^{\mathrm{d}}$ & $0.62 \pm 0.07^{\mathrm{a}}$ & $0.31 \pm 0.04^{\mathrm{cd}}$ & $0.40 \pm 0.05^{\mathrm{b}}$ & $0.30 \pm 0.01^{\mathrm{d}}$ & $0.42 \pm 0.01^{b}$ & $0.37 \pm 0.03^{b c}$ \\
\hline $20: 5 n-3$ & 0.87 & $2.08 \pm 0.10^{\mathrm{a}}$ & $0.59 \pm 0.04^{\mathrm{e}}$ & $0.92 \pm 0.05^{\mathrm{d}}$ & $1.17 \pm 0.11^{\mathrm{c}}$ & $1.47 \pm 0.01^{\mathrm{b}}$ & $0.84 \pm 0.01^{\mathrm{d}}$ & $1.19 \pm 0.10^{\mathrm{c}}$ \\
\hline $22: 5 n-3$ & 0.25 & $0.59 \pm 0.05^{\mathrm{a}}$ & $0.21 \pm 0.02^{\mathrm{e}}$ & $0.26 \pm 0.04^{\mathrm{de}}$ & $0.38 \pm 0.02^{\mathrm{c}}$ & $0.46 \pm 0.04^{\mathrm{b}}$ & $0.28 \pm 0.04^{\mathrm{d}}$ & $0.35 \pm 0.02^{\mathrm{c}}$ \\
\hline $22: 6 n-3$ & 4.76 & $10.73 \pm 0.40^{\mathrm{a}}$ & $5.04 \pm 0.19^{f}$ & $5.72 \pm 0.39^{\mathrm{e}}$ & $8.00 \pm 0.32^{\mathrm{c}}$ & $8.60 \pm 0.18^{b}$ & $5.65 \pm 0.47^{\mathrm{e}}$ & $6.62 \pm 0.35^{\mathrm{d}}$ \\
\hline PUFA n-3 & 13.28 & $16.67 \pm 0.52^{\mathrm{e}}$ & $11.43 \pm 0.04^{\mathrm{g}}$ & $27.57 \pm 0.72^{\mathrm{a}}$ & $14.19 \pm 0.45^{\mathrm{f}}$ & $22.51 \pm 0.22^{b}$ & $18.77 \pm 0.40^{\mathrm{c}}$ & $17.91 \pm 0.69^{\mathrm{d}}$ \\
\hline HUFA n-3 & 5.63 & $12.80 \pm 0.45^{\mathrm{a}}$ & $5.63 \pm 0.22^{\mathrm{f}}$ & $6.64 \pm 0.42^{\mathrm{e}}$ & $9.18 \pm 0.43^{\mathrm{c}}$ & $10.07 \pm 0.19^{b}$ & $6.49 \pm 0.48^{\mathrm{e}}$ & $7.81 \pm 0.44^{\mathrm{d}}$ \\
\hline PUFA & 16.68 & $19.27 \pm 0.66^{\mathrm{e}}$ & $14.06 \pm 0.17^{\mathrm{g}}$ & $30.75 \pm 0.57^{\mathrm{a}}$ & $16.71 \pm 0.58^{f}$ & $25.26 \pm 0.24^{b}$ & $21.82 \pm 0.58^{\mathrm{c}}$ & $20.69 \pm 0.72^{\mathrm{d}}$ \\
\hline PUFA/SFA & 0.90 & $0.69 \pm 0.04^{\mathrm{e}}$ & $0.62 \pm 0.02^{f}$ & $1.41 \pm 0.02^{\mathrm{a}}$ & $0.71 \pm 0.04^{\mathrm{e}}$ & $1.04 \pm 0.02^{\mathrm{b}}$ & $0.96 \pm 0.03^{\mathrm{c}}$ & $0.89 \pm 0.02^{\mathrm{d}}$ \\
\hline$n-6 / n-3$ & 0.26 & $0.16 \pm 0.02^{\mathrm{bc}}$ & $0.23 \pm 0.02^{\mathrm{a}}$ & $0.12 \pm 0.01^{\mathrm{d}}$ & $0.18 \pm 0.01^{\mathrm{b}}$ & $0.12 \pm 0.00^{\mathrm{d}}$ & $0.16 \pm 0.01^{b c}$ & $0.15 \pm 0.01^{\mathrm{c}}$ \\
\hline
\end{tabular}

See Table 1 for diet abbreviations.

Values in the same row with the same superscripts are not significantly different $(P>0.05)$. 
Table 6. Correlation between dietary fatty acid concentrations and fatty acid concentrations in fillet of rainbow trout fed the experimental diets for 8 weeks.

\begin{tabular}{|c|c|c|}
\hline Fatty acids & Correlation coefficient $(r)$ & Slope \\
\hline $16: 0$ & 0.93 & 0.50 \\
\hline $18: 0$ & 0.91 & 0.36 \\
\hline SFA & 0.89 & 0.45 \\
\hline $18: 1 n-9$ & 0.96 & 0.75 \\
\hline MUFA & 0.96 & 0.82 \\
\hline $18: 2 n-6$ & 0.62 & 0.75 \\
\hline $20: 4 n-6$ & 0.47 & -0.73 \\
\hline PUFA n-6 & 0.86 & -0.45 \\
\hline $18: 3 n-3$ & 0.99 & 0.91 \\
\hline $20: 5 n-3$ & 0.87 & 0.53 \\
\hline $22: 6 n-3$ & 0.86 & 1.07 \\
\hline PUFA n-3 & 0.96 & \\
\hline PUFA & 0.96 & \\
\hline
\end{tabular}

\section{Discussion}

The results of the present study suggest that canola and flaxseed oils can be used to replace fish oil without adverse effects on growth performance of rainbow trout fingerlings, as reported in other studies [33-36]. This was evident by the weight gain and feed conversion ratio of fish fed dietary treatments which ranged from $224.56 \pm 17.82 \%$ to $260.09 \pm 4.25 \%$ and $0.90 \pm 0.17$ to $1.07 \pm 0.41$, respectively, with no significant differences from fish fed all experimental diets.

In agreement with previous studies of similar nature $[21,33,35,37,38]$, considerable differences were evident in the fatty acid composition of fish fed different lipid sources. For example, there was a high increase in the levels of linoleic and $\alpha$-linolenic acids in fish fed COD and FxOD, respectively.

As reported by other researchers [15,16,23,35,39-42], a high correlations are also exist between the individual fatty acids as well as MUFA and PUFA of a diet and the fish fillet (Table 6). There was, however, a high correlation between the amount of SFA in the diet and SFA in the fillet, which was not in accordance with the findings of Turchini et al. $(2003 a, b)[35,40]$ who postulated that SFA were not used efficiently by Murray cod (Maccullochellapeeliipeelii) as an energy source and were subsequently deposited at an optimal level in preference to the other major fatty acid classes.

It is well known that freshwater fish have a dietary requirement for $n-3$ and n- 6 fatty acids, predominantly in the form of $\alpha$-linolenic acid and linoleic acid [23,24,33,43-45]. In comparison to marine fish species, freshwater fish are also generally better equipped to desaturate and elongate these base fatty acids to higher homologs [23,45]. This study observed $\alpha$-linolenic acid in lower concentrations in the muscle than in the diets. It is therefore suspected that a high degree of metabolism of this fatty acid for $\beta$-oxidation and/or desaturation and elongation is taking place in fingerlings of rainbow trout. This is further bolstered by the presence of $n-3$ desaturation and elongation enzyme products in the form of 18:4n-3 and 20:3n-3 in fish fed the FxOD, FFxOD and FCFxOD. These fatty acids were found in much lower concentrations in the diets. Likewise, fish fed the COD, FCOD and FCFxOD contained n- 6 desaturation and elongation intermediates (18:3n-6 and 20:3n-6) and indicate an elongation and desaturation of linoleic acid via $\Delta 6$ desaturase. However, the further desaturation of $20: 3 n-6$ to 20:4n-6 and 20:3n-3 to EPA and ultimately DHA was shrouded by high concentrations of these fatty acids within the fillet of initial fish samples. The Department of Health of England [46] recommends a minimum PUFA/SFA ratio of 0.45 , and a maximum $n-6 / n-3$ of 4.0 . Table 5 shows that our fish in all treatments met the PUFA/SFA and n-6/n-3 ratios. Despite the decrease in EPA and DHA in fillet from fish fed canola and flaxseed oils, the trout fillets remain a relatively rich source of these fatty acids with a $200 \mathrm{~g}$ serving of the fillets from fish fed FCOD and FFxOD diets supplying 704 and $584 \mathrm{mg}$ of EPA plus DHA, respectively. This meets the intake of $500 \mathrm{mg} /$ day of EPA plus DHA recommended by the International Society for the Study of Fatty Acids and Lipids [47].

\section{Conclusions}

Present study showed the substitution of fish oil with canola and flaxseed oils in the rainbow trout diet have been possible without any negative effects on the growth and feed conversion ratio. However, the reflection of the dietary oil source on the fillet fatty acid composition of the fish could be a potential drawback for vegetable oil substitution from a human nutritional point of view, given the decreases in levels of EPA and DHA in fish fed the vegetable oil diets. Further investigation into the benefits of other vegetable oils or indeed a blend of various vegetable oils is required in order to reduce usage of traditionally used fish oils, while simultaneously avoiding a reduction in the human health 
protective properties found within fish flesh.

\section{Acknowledgements}

This study was funded $(1,500 \$)$ by the Isfahan University of Technology. The authors thank E. Mottaghi and B. Bahrami for technical assistance throughout the project.

\section{REFERENCES}

[1] New, M. 1999. Global aquaculture: current trends of challenges for the 21st century. World Aquaculture 30:8-13.

[2] Jacobs, M. N., J. Ferrario, and C. Byrne. 2002a. Investigation of polychlorinated dibenzo-p-dioxins dibenzo-p-furans and selected coplanar biphenyls in Scottish farmed Atlantic salmon (Salmosalar). Chemosphere 47:183-191.

[3] Jacobs, M. N., A. Covaci, and P. Schepens. 2002b. Investigation of selected persistant organic pollutants in farmed Atlantic salmon (Salmosalar), salmon aquaculture feed, and fish oil components of the feed. Environmental Science and Technology 36:2797-2805.

[4] Hites, R. A., J. A. Foran, D. O. Carpenter, M. C. Hamilton, B. A. Knuth, and S. J. Schwager. 2004a, Global assessment of organic contaminants in farmed salmon. Science 303:226-229.

[5] Hites, R. A., J. A. Foran, S. J. Schwager, B. A. Knuth, M.C. Hamilton, and D. O. Carpenter. 2004b. Global assessment of polybrominateddiphenyl ethers in farmed and wild salmon. Environmental Science and Technology 38:4945-4949.

[6] Birnbaum, L. S., and J. Tuomisto. 2000. Non-carcinogenic effects of TCDD in animals. Food Additives and Contaminants 17:275-288.

[7] Baccarelli, A., P. Mocarelli, D. G. Patterson, M. Bonzini, A. D. Pesatori, N. Caporaso, and M. T. Landi. 2002. Immunologic effects of dioxin: new results from Seveso and comparison with other studies. Environmental Health Perspectives 110:1169-1173.

[8] Van Den Heuvel, R. L., G. Koppen, J. A. Staessen, E. D. Hond, G. Verheyen, T. S. Nawrot, H. A. Roels, R. Vleietinck, and G. E. R. Schoeters. 2002. Immunologic biomarkers in relation to exposure markers of PCBs and dioxins in Flemish adolescents (Belgium). Environmental Health Perspectives 110:595-600.

[9] Fritsche, K. L., and P. V. Johnston. 1990. Effect of dietary a-linolenic acid on growth, metastasis, fatty acid profile and prostaglandin production of two marine mammary adenocarcinomas. Journal of Nutrition 120:1601-1609.

[10] Kubow, S. 1993. Lipid oxidation products in foods and atherogenesis. Nutrition Reviews 51:33-40.

[11] Waagbø, R., K. Sandnes, O. J. Torrissen, A. Sandvin, and Ø. Lie. 1993. Chemical and sensory evaluation of fillets from Atlantic salmon (Salmosalar) fed three different levels of n-3 polyunsaturated fatty acids at two levels of vitamin E. Food Chemistry 46:361-366.
[12] NRC (National Research Council). 1993. Nutrient Requirement of Fish. National Academy Press, Washington, DC.

[13] Bell, J. G., R. J. Henderson, D. R. Tocher, F. McGhee, J. R. Dick, A. Porter, R. Smullen, and J. R. Sargent. 2002. Substituting fish oil with crude palm oil in the diet of Atlantic salmon (Salmosalar) affects tissue fatty acid compositions and hepatic fatty acid metabolism. Journal of Nutrition 132:222-230.

[14] Bell, J. G., D. R. Tocher, R. J. Henderson, J. R. Dick, and V. O. Crampton. 2003a. Altered fatty acid compositions in Atlantic salmon (Salmosalar) fed diets containing linseed and rapeseed oils can be partially restored by a subsequent fish oil finishing diet. Journal of Nutrition 133:2793-2801.

[15] Chen, Y. C., J. Nguyen, K. Semmens, S. Beamer, and J. Jaczynski. 2006. Enhancement of omega-3 fatty acid content in rainbow trout (Oncorhynchusmykiss) fillets. Journal of Food Science 71:383-389.

[16] Simmons, C. A., P. Turk, S. Beamer, J. Jaczynski, K. Semmens, and K. E. Matak. 2011. The effect of a flaxseed oil-enhanced diet on the product quality of farmed brook trout (Salvelinusfontinalis) fillets. Journal of Food Science 76:192-197.

[17] Henderson, R. J., and D. R. Tocher. 1987. The lipid composition and biochemistry of freshwater fish. Progress in Lipid Research 26:281-347.

[18] Lauritzen, L., H. S. Hansen, M. H. Jorgensen, and K. F. Michaelsen. 2001. The essentiality of long chain n-3 fatty acids in relation to development and function of the brain and retina. Progress in Lipid Research 40:1-94.

[19] Wonnacott, E. J., R. L. Lane, and C. C. Kohler. 2004. Influence of dietary replacement of menhaden oil with canola oil on fatty acid composition of sunshine bass. North American Journal of Aquaculture 66:243-250.

[20] Subhadra, B., R. Lochmann, S. Rawles, and R. G. Chen. 2006. Effect of dietary lipid source on the growth, tissue composition and hematological parameters of largemouth bass (Micropterussalmoides). Aquaculture 255:210-222.

[21] Caballero, M. J., A. Obach, G. Rosenlund, D. Montero, M. Gisvold, and M. S. Izquierdo. 2002. Impact of different dietary lipid sources on growth, lipid digestibility, tissue fatty acid composition and histology of rainbow trout, Oncorhynchusmykiss. Aquaculture 214:253-271.

[22] Rosenlund, G., A. Obach, M. G. Sandberg, H. Standal, and K. Tveit. 2001. Effect of alternative lipid sources on long-term growth performance and quality of Atlantic salmon (Salmosalar). Aquaculture Research 32:323-328.

[23] Guillou, A., P. Soucy, M. Khalil, and L. Adambounou. 1995. Effects of dietary vegetable and marine lipid on growth, muscle fatty acid composition and organoleptic quality of flesh of brook charr (Salvelinusfontinalis). Aquaculture 136:351-362.

[24] Izquierdo, M. S., A. Obach, L. Arantzamendi, D. Montero, L. Robaina, and G. Rosenlund. 2003. Dietary lipid sources for sea bream and sea bass: growth performance, tissue composition and flesh quality. Aquaculture Nutrition 9:397-407.

[25] Greene, D. H. S., and D. P. Selivonchick. 1990. Effects of 
dietary vegetable, animal and marine lipids on muscle lipid and hematology of rainbow trout (Oncorhynchusmykiss). Aquaculture 89:165-182.

[26] Abery, N. W., R. M. Gunasekera, and S. S. De Silva. 2002. Growth and nutrient utilization of Murray cod (Maccullochellapeeliipeelii) fingerlings fed diets with varying levels of soybean meal and blood meal. Aquaculture Research 33:279-289.

[27] De Silva, S., R. Gunasekera, R. Collins, and B. Ingram. 2002. Performance of juvenile Murray cod, Maccullochellapeeliipeelii, fed with diets of different protein to energy ratio. Aquaculture Nutrition 8:79-85.

[28] AOAC (Association of Official Analytical Chemists). 1990. Official methods of analysis, 15th edition. Association of Official Analytical Chemists, Arlington, Virginia, USA.

[29] Folch, J. M., M. Lees, and G. H. Sloane-Stanley. 1957. A simple method for the isolation and purification of total lipids from animal tissues. Journal of Biological Chemistry 226:497-509.

[30] Ways, P., and D. J. Hanahan. 1964. Characterization and quantification of red cell lipids in normal man. Journal of Lipid Research 5:318-328.

[31] Christie, W. W., editor. 1982. Lipid analysis. Pergamon Press, Oxford.

[32] Tocher, D. R., and D. G. Harvie. 1988. Fatty acid compositions of the major phosphoglycerides from fish neural tissues: (n-3) and (n-6) polyunsaturated fatty acids in rainbow trout (Salmogairdneri) and cod (Gadusmorhua) brains and retinas. Fish Physiology and Biochemistry 5:229-239.

[33] Martino, R. C., J. E. P. Cyrino, L. Portz, and L. C. Trugo. 2002. Performance and fatty acid composition of surubim (Pseudoplatystomacoruscans) fed diets with animal and plant lipids. Aquaculture 209:233-246.

[34] Regost, C., J. Arzel, J. Robin, G. Rosenlund, and S. J. Kaushik. 2003. Total replacement of fish oil by soybean or linseed oil with a return to fish oil in turbot (Psetta maxima), Growth performance, flesh fatty acid profile, and lipid metabolism. Aquaculture 217:465-482.

[35] Turchini, G. M., T. Mentasti, L. Frøyland, E. Orban, F. Caprino, V. M. Moretti, and F. Valfre. 2003b. Effects of alternative dietary lipid sources on performance, tissue chemical composition, mitochondrial fatty acid oxidation capabilities and sensory characteristics in brown trout (Salmotrutta). Aquaculture 225:251-267.

[36] Karayücel, İ., and S. Dernekbaşi. 2010. Effect of dietary canola oil level on growth, feed utilization, and body composition of rainbow trout OncorhynchusmykissL.. The Israeli Journal of Aquaculture-Bamidgeh, 62:155-162.
[37] Glencross, B., W. Hawkins, and J. Curnow. 2003. Evaluation of canola oils as alternative lipid resources in diets for juvenile red sea bream, Pagrusauratus. Aquaculture Nutrition 9:305-315.

[38] Dernekbaşi, S., İ. Karayücel, and A. Öksüz. 2011. Effect of dietary canola oil level on fatty acid composition of rainbow trout OncorhynchusmykissL..The Israeli Journal of Aquaculture-Bamidgeh, IIC:63:535-545.

[39] Bell, J. G., F. McGhee, P. J. Campbell, and J. R. Sargent. 2003b. Rapeseed oil as an alternative to marine fish oil in diets of post-smolt Atlantic salmon (Salmosalar): changes in flesh fatty acid composition and effectiveness of subsequent fish oil "wash out". Aquaculture 218:515-528.

[40] Turchini, G. M., R. M. Gunasekera, and S. S. De Silva. 2003a. Effect of crude oil extracts from trout offal as a replacement for fish oil in the diets of the Australian native fish Murray cod, Maccullochellapeeliipeelii. Aquaculture Research 34:697-708.

[41] Torstensen, L., L. Frøyland, and O. Lie. 2004. Replacing dietary fish oil with increasing levels of rapeseed oil and olive oil, effects on Atlantic salmon (Salmosalar) tissue and lipoprotein composition and lipogenic enzyme activities. Aquaculture Nutrition 10:175-192.

[42] Chen, Y. C., J. Nguyen, K. Semmens, S. Beamer, and J. Jaczynski. 2008. Chemical changes in omega-3-enhanced farmed rainbow trout (Oncorhynchusmykiss) fillets during abusive-temperature storage. Food Control 19:599-608.

[43] Kanazawa, A., S. I. Teshima, and K. Ono. 1979. Relationship between essential fatty acid requirements of aquatic animals and the capacity for bioconversion of linolenic acid to highly unsaturated fatty acids. Comparative Biochemistry and Physiology 63B:295-298.

[44] Kanazawa, A., S. I. Teshima, M. Sakamoto, and M. Awal. 1980. Requirements of Tilapia zilli for essential fatty acids. Bulletin of the Japanese Society of Scientific Fisheries 46:1353-1356.

[45] Tocher, D. R. 2003. Metabolism and functions of lipids and fatty acids in teleost fish. Reviews of Fish Science 11:107-184.

[46] HMSO. 1994. Department of health of england. nutritional aspects of cardiovascular disease. Report on Health and Social Subjects n. 46. HMSO, London.

[47] Simopoulos, A. P., A. Leaf, and N. Salem. 1999. Workshop on the essentiality of and recommended dietary intakes for omega- 6 and omega-3 fatty acids. International Society for the Stu 